\title{
Publisher Correction: Organic reactivity from mechanism to machine learning
}

Kjell Jorner(D, Anna Tomberg (D, Christoph Bauer(D, Christian Sköld@ and Per-Ola Norrby (1)

Correction to: Nature Reviews Chemistry https://doi.org/10.1038/s41570-021-00260-x, published online 16 March 2021.

In the version originally published, the sentence beginning "Reactivity models often focus on" contained a typographical error and should have read "Reactivity models often focus on reaction barriers". The original article has been corrected.

https://doi.org/10.1038/s41570-021-00272-7 I Published online 22 March 2021

(๑) Springer Nature Limited 2021 